\title{
Meta-analysis demonstrates lack of an association of microsomal epoxide hydrolase 1 polymorphisms with esophageal cancer risk
}

\author{
J.J. Hu ${ }^{1 *}$, Z.T. Wang ${ }^{2 *}$ and B. $\mathbf{L i}^{1}$ \\ ${ }^{1}$ Department of Nuclear Medicine, Ruijin Hospital, School of Medicine, \\ Shanghai Jiaotong University, Shanghai, China \\ ${ }^{2}$ Department of Gastroenterology, Ruijin Hospital, School of Medicine, \\ Shanghai Jiaotong University, Shanghai, China \\ *These authors contributed equally to this study. \\ Corresponding author: B. Li \\ E-mail: mellejj@hotmail.com
}

Genet. Mol. Res. 12 (4): 4540-4548 (2013)

Received September 24, 2012

Accepted March 11, 2013

Published October 15, 2013

DOI http://dx.doi.org/10.4238/2013.October.15.2

\begin{abstract}
Epoxide hydrolases metabolize exogenous chemicals, including carcinogens such as polycyclic aromatic hydrocarbons. The relationship between microsomal epoxide hydrolase 1 (EPHX1) polymorphisms and esophageal cancer risk has been investigated in various ethnic populations, but the results have been contradictory. We investigated the association of EPHX1 Tyr113His and His139Arg polymorphisms with esophageal cancer via a comprehensive metaanalysis. Publications before August 20, 2012 were included. Eight studies concerning Tyr113His polymorphism associated with 1158 esophageal cancer cases and 1868 controls were identified; 7 studies concerning association of His139Arg with 901 esophageal cancer cases and 1615 controls were also included. A random-effect model was applied, irrespective of between-study heterogeneity. Data and study quality were assessed in duplicate. No significant association was found in either the allele or genotype models for Tyr113His or His139Arg
\end{abstract}


polymorphism with risk for esophageal cancer. Lack of association was also identified in stratified analyses by ethnicity. No publication bias was observed. We conclude that current evidence does not demonstrate association of EPHX1 Tyr113His or His139Arg polymorphisms with risk for development of esophageal cancer.

Key words: Esophageal cancer; $E P H X 1$ gene; Meta-analysis; Polymorphism

\section{INTRODUCTION}

Esophageal cancer (EC) is one of the most common malignancies in the world, especially in the regions called "the esophageal cancer belt", which stretches from NorthernCentral China westward through Central Asia to Northern Iran (Xing et al., 2003). The development of $\mathrm{EC}$ is a multifactorial process involving a variety of risk factors. Cumulative evidence suggests that smoking, drinking, micronutrient deficiency, and dietary carcinogen exposure may cause this disease. However, only a small portion of the at-risk population exposed to the above factors will develop EC, while many EC cases involve individuals without those known risk factors (Hiyama et al., 2007), indicating that genetic factors may play a part in esophageal carcinogenesis.

Microsomal epoxide hydrolase, EPHX1, also known as $\mathrm{mEH}$, which is one of the phase I metabolism enzymes, plays a dual role both in detoxication and activation of procarcinogens (Hassett et al., 1994a). On the one hand, it catalyzes the hydrolysis of arene, alkene, and aliphatic epoxides from polycyclic aromatic hydrocarbons and aromatic amines to less reactive and more water-soluble dihydrodiols (Lancaster et al., 1996; Harrison et al., 1999); whereas on the other hand, it activates some polycyclic aromatic hydrocarbons into a more carcinogenic form (Sims et al., 1974). Therefore, the role of EPHX1 in carcinogenesis may depend on exposure to different environmental substrates.

The EPHX1 gene consists of 9 exons and 8 introns and is located on chromosome 1q42. Functional studies have shown that there are 2 polymorphic sites in the gene affecting EPHX1 enzyme activity (Hassett et al., 1994b). A tyrosine to histidine substitution in exon 3 (Tyr113His) of the EPHX1 gene decreases in vitro enzyme activity by $40 \%$, whereas a histidine to arginine substitution in exon 4 (His139Arg) increases in vitro enzyme activity by $25 \%$ (Hassett et al., 1994a). Variations in enzyme activity of EPHX1 as a result of such polymorphisms may lead to interindividual variations in susceptibility to chemical carcinogen-induced cancers, including EC. Although some studies have attempted to link EPHX1 gene Tyr113His and His139Arg polymorphisms to EC, the resulting data are not often reproducible (Zhang et al., 2003; Casson et al., 2003, 2006; Lin et al., 2006; Wang et al., 2003, 2006; Jain et al., 2008; Ihsan et al., 2010; Dura et al., 2012). As a caveat, failure of replication may result from the genetic heterogeneity between different races or ethnicities, as well as individual studies with relatively low statistical power. To systematically address this issue, we decided to investigate the association of EPHXI gene Tyr113His and His139Arg polymorphisms with occurrence of EC and whether the polymorphisms show genetic heterogeneity across different continental regions worldwide. Robustness was tested by publication bias. 


\section{MATERIAL AND METHODS}

\section{Literature search}

We conducted a comprehensive literature search from 4 widely used databases, namely MEDLINE, EMBASE, ISI Web of Knowledge, and China National Knowledge Infrastructure, up to August 20, 2012, using a combination of key words 'esophageal cancer' and ' $E P H X I$ '. As a prerequisite, all reports were written in English and performed in humans. Reference lists of reviews and retrieved papers were also screened for citations of relevant articles that were not initially identified. If there were more than one publication from the same study population, we selected the study with the most complete and recent results.

\section{Inclusion/exclusion criteria}

Studies identified satisfied the following criteria: 1) evaluation of at least one studied polymorphism (Tyr113His and/or His139Arg) in the EPHX1 gene and the risk for EC; 2) casecontrol or cross-sectional or nested case-control study in design; 3) availability of genotype or allele counts of studied polymorphisms between patients and controls to estimate odds ratio (OR) and its corresponding 95\% confidence interval (CI). We excluded studies for lacking the necessary information on the cases' and controls' alleles or genotypes. Meanwhile, we merely focused on EC other than other second neoplasms.

\section{Extracted information}

Two authors (J.J. Hu and Z.T. Wang) independently extracted the following information from all eligible studies: first author's last name, year of publication, ethnicity of the population studied, study design, number of subjects in each category, baseline characteristics of the study population, and the number of individuals in both case-patient and control group within each genotype tested. Besides, information on Hardy-Weinberg equilibrium test was also tracked or calculated manually if unavailable. Any encountered discrepancies were adjudicated by a discussion and a $100 \%$ consensus was reached.

\section{Statistical analysis}

In this meta-analysis, we assessed the association of EPHX1 gene Tyr113His and His139Arg polymorphisms with EC risk in allelic, homozygous and dominant and recessive models, respectively. Crude OR and $95 \% \mathrm{CI}$ were calculated to compare contrasts of alleles or genotypes between patients and controls.

Deviation from Hardy-Weinberg equilibrium was assessed using the Pearson $\chi^{2}$ test. The random-effects model using the DerSimonian and Laird method was employed to bring the individual effect-size estimates together irrespective of between-study heterogeneity (Cohn and Becker, 2003). Heterogeneity was evaluated by the $\mathrm{I}^{2}$ statistic, which was documented for the percentage of the observed between-study variability due to heterogeneity rather than chance with its values ranging from 0 to $100 \%\left(\mathrm{I}^{2}=0-25 \%\right.$, no heterogeneity; $\mathrm{I}^{2}=25-50 \%$, moderate heterogeneity; $\mathrm{I}^{2}=50-75 \%$, large heterogeneity; $\mathrm{I}^{2}=75-100 \%$, extreme heterogene- 
ity) (Higgins and Thompson, 2002; Higgins et al., 2003). In the case of between-study heterogeneity, we examined the study characteristics that can stratify the studies into subgroups with homogeneous effects. Subgroup analyses were conducted after stratifying studies and were performed on various ethnic/geographic populations.

Finally, evidence for publication bias was assessed using the Egger test and visual funnel plot inspection. The Egger test detects funnel plot asymmetry by determining whether the intercept deviates significantly from zero in a regression of the standardized effect estimates against their precision.

Probability less than 0.05 was judged significant with the exception of the $\mathrm{I}^{2}$ statistic and publication tests, where a significance level of less than 0.1 was chosen. Statistical analyses were performed using STATA version 11.0 for Windows.

\section{RESULTS}

\section{Description of available studies}

According to the search rules, a total of 22 articles were initially identified. Further application of our inclusion/exclusion criteria left 9 articles (Zhang et al., 2003; Casson et al., 2003, 2006; Lin et al., 2006; Wang et al., 2003, 2006; Jain et al., 2008; Ihsan et al., 2010; Dura et al., 2012), including 8 studies for the Tyr113His polymorphism with 1158 EC cases and 1868 controls (Wang et al., 2003; Zhang et al., 2003; Casson et al., 2003, 2006; Lin et al., 2006; Jain et al., 2008; Ihsan et al., 2010; Dura et al., 2012), and 7 studies for His139Arg with 901 EC and 1615 controls (Wang et al., 2003; Casson et al., 2003, 2006; Lin et al., 2006; Jain et al., 2008; Ihsan et al., 2010; Dura et al., 2012). The detailed selection process and the baseline characteristics of the qualified studies are presented in Figure 1 and Table 1, respectively.

\section{Tyr113His polymorphism and EC}

Overall null significant association was found for the Tyr113His polymorphism in allele comparison $(\mathrm{OR}=0.88,95 \% \mathrm{CI}=0.73-1.06 ; \mathrm{P}=0.178)$, homozygote $(\mathrm{OR}=0.87,95 \% \mathrm{CI}$ $=0.64-1.19, \mathrm{P}=0.384)$, dominant model $(\mathrm{OR}=0.81,95 \% \mathrm{CI}=0.66-1.01, \mathrm{P}=0.061)$, and recessive model $(\mathrm{OR}=0.94 ; 95 \% \mathrm{CI}=0.68-1.29 ; \mathrm{P}=0.697)($ Table 2$)$.

Considering the fact that ethnicity differences may bias the overall estimates, we therefore conducted separate analyses based on ethnicity, where we classified 8 study populations into Chinese (Wang et al., 2003; Zhang et al., 2003; Lin et al., 2006), Caucasians (Casson et al., 2003, 2006; Dura et al., 2012), and Indians (Jain et al., 2008; Ihsan et al., 2010). As shown in Table 2, albeit nonsignificant, comparison of $113 \mathrm{Tyr}$ versus 113 His generated a weakly protective tendency for $\mathrm{EC}$ in Chinese $(\mathrm{OR}=0.87 ; 95 \% \mathrm{CI}=0.59-1.29 ; \mathrm{P}=0.495)$, Caucasians $(\mathrm{OR}=0.86 ; 95 \% \mathrm{CI}=0.57-1.30 ; \mathrm{P}=0.477)$, and Indians $(\mathrm{OR}=0.83 ; 95 \% \mathrm{CI}=0.57-1.22 ; \mathrm{P}$ $=0.352$ ), Similar tendencies were noted for other genetic models except for Caucasians in the recessive model $(\mathrm{OR}=1.01 ; 95 \% \mathrm{CI}=0.52-1.98 ; \mathrm{P}=0.976)($ Table 2$)$.

\section{His139Arg polymorphism and EC}

Still, there was no significant association between the His139Arg polymorphism and 
$\mathrm{EC}$ risk in allele comparison $(\mathrm{OR}=0.90,95 \% \mathrm{CI}=0.72-1.13 ; \mathrm{P}=0.379)$, homozygote $(\mathrm{OR}=$ $0.67,95 \% \mathrm{CI}=0.33-1.36, \mathrm{P}=0.270)$, dominant model $(\mathrm{OR}=0.98,95 \% \mathrm{CI}=0.68-1.41, \mathrm{P}=$ $0.911)$, and recessive model $(\mathrm{OR}=0.85 ; 95 \% \mathrm{CI}=0.60-1.22 ; \mathrm{P}=0.379)$. Lack of association was also identified in analyses stratified by ethnicity.

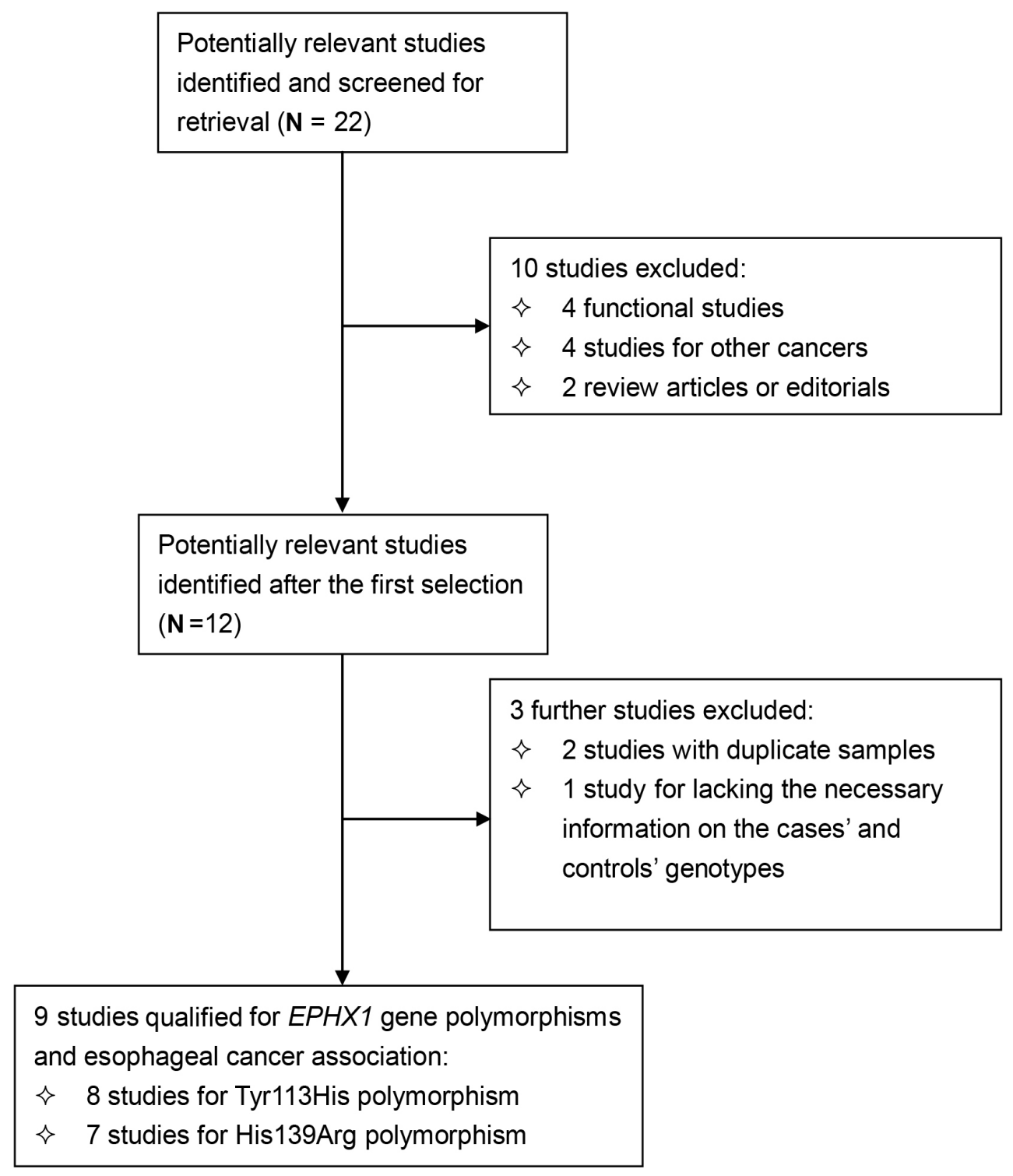

Figure 1. Flow diagram of search strategy and study selection 


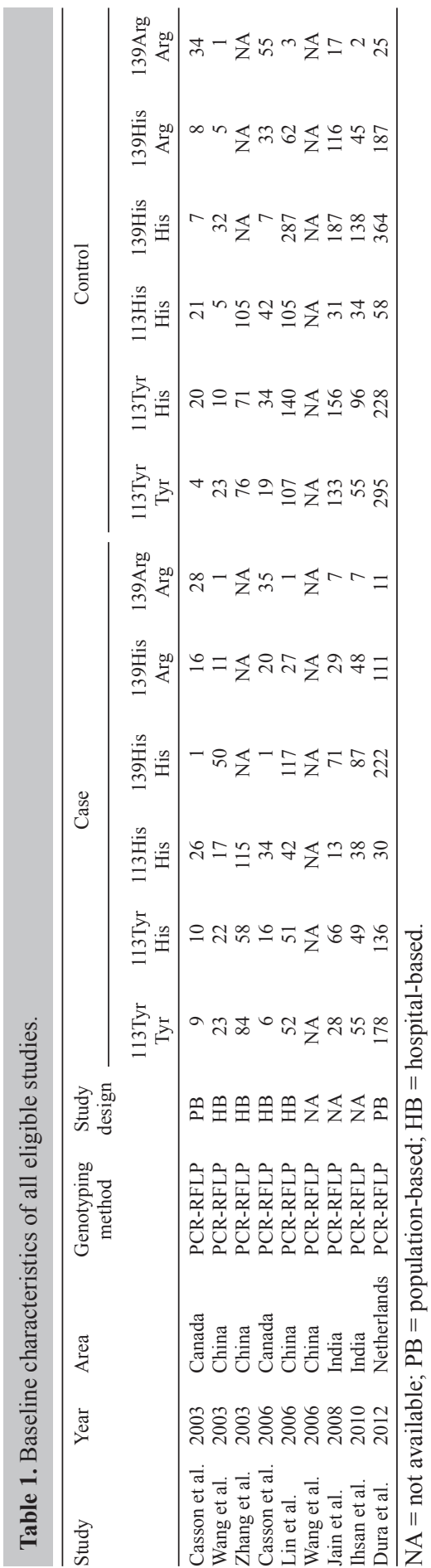


Table 2. Subgroup analysis of EPHX1 gene Tyr113His and His139Arg polymorphisms and esophageal cancer.

\begin{tabular}{|c|c|c|c|c|c|c|c|c|}
\hline \multirow[t]{2}{*}{ Variables } & \multicolumn{2}{|l|}{ Allele } & \multicolumn{2}{|c|}{ Homozygote } & \multicolumn{2}{|c|}{ Dominant model } & \multicolumn{2}{|c|}{ Recessive model } \\
\hline & OR $(95 \% \mathrm{CI})$ & $\mathrm{I}^{2}$ & OR $(95 \% \mathrm{CI})$ & $\mathrm{I}^{2}$ & OR $(95 \% \mathrm{CI})$ & $\mathrm{I}^{2}$ & OR $(95 \% \mathrm{CI})$ & $\mathrm{I}^{2}$ \\
\hline \multicolumn{9}{|c|}{ Tyr113His polymorphism } \\
\hline Total & $0.88(0.73-1.06)$ & $59.9 \%$ & $0.87(0.64-1.19)$ & $43.0 \%$ & $0.81(0.66-1.01)$ & $18.2 \%$ & $0.94(0.68-1.29)$ & $68.3 \%$ \\
\hline \multicolumn{9}{|c|}{ Descent of populations } \\
\hline Caucasian & $0.86(0.57-1.30)$ & $62.6 \%$ & $0.93(0.43-2.02)$ & $55.0 \%$ & $0.77(0.45-1.33)$ & $54.2 \%$ & $1.01(0.52-1.98)$ & $53.7 \%$ \\
\hline Chinese & $0.87(0.59-1.29)$ & $74.1 \%$ & $0.89(0.52-1.53)$ & $59.6 \%$ & $0.89(0.64-1.22)$ & $22.2 \%$ & $0.93(0.55-1.57)$ & $69.9 \%$ \\
\hline Indian & $0.83(0.57-1.22)$ & $66.4 \%$ & $0.71(0.40-1.23)$ & $26.9 \%$ & $0.67(0.44-1.02)$ & $0.0 \%$ & $0.87(0.29-2.54)$ & $90.3 \%$ \\
\hline \multicolumn{9}{|c|}{ His139Arg polymorphism } \\
\hline Total & $0.90(0.72-1.13)$ & $42.8 \%$ & $0.67(0.33-1.36)$ & $36.1 \%$ & $0.98(0.68-1.41)$ & $0.0 \%$ & $0.85(0.60-1.22)$ & $57.0 \%$ \\
\hline \multicolumn{9}{|c|}{ Descent of populations } \\
\hline Caucasian & $1.01(0.82-1.24)$ & $0.0 \%$ & $0.50(0.11-2.33)$ & $62.4 \%$ & $1.12(0.73-1.72)$ & $0.0 \%$ & $0.44(0.10-1.87)$ & $63.1 \%$ \\
\hline Chinese & $0.94(0.62-1.43)$ & $0.0 \%$ & $1.35(0.23-7.89)$ & $0.0 \%$ & $1.39(0.24-8.10)$ & $0.0 \%$ & $0.92(0.59-1.43)$ & $0.0 \%$ \\
\hline Indian & $0.82(0.37-1.80)$ & $87.2 \%$ & $0.47(0.10-2.27)$ & $67.2 \%$ & $0.48(0.13-1.73)$ & $51.8 \%$ & $0.87(0.34-2.22)$ & $87.7 \%$ \\
\hline
\end{tabular}

$\mathrm{OR}=$ odds ratio $; \mathrm{CI}=$ confidence interval.

\section{Publication bias}

As reflected by the funnel plot and the Egger test, there was a low probability of publication bias for the EPHX1 gene Tyr113His $(\mathrm{P}=0.134)$ and His139Arg $(\mathrm{P}=0.170)$ polymorphisms.

\section{DISCUSSION}

To the best of our knowledge, this is the first meta-analysis assessing the association of the EPHX1 gene Tyr113His and His139Arg polymorphisms with EC. Although some statistical biases could not be eliminated and as there was evidence of between-study heterogeneity, our results suggested that $E P H X 1$ gene Tyr113His and His139Arg polymorphisms may not be potential candidates for the development of EC, while ethnicity was identified as a potentially significant source of between-study heterogeneity in this meta-analysis. Nonetheless, there was a low probability of publication bias for all allelic/genotypic comparisons, indicating the robustness of our results.

Genetic heterogeneity is an inevitable problem in any disease identification strategy (Hemminki et al., 2006). As shown in this study, we speculated that the EPHX1 gene Tyr113His and His139Arg polymorphisms may have different roles across different ethnic populations. On the one hand, there were striking differences in terms of 113 Tyr and 139His allele frequency in patients between Canadians and Chinese (27.72 vs 48.38\% in 113Tyr, 19.80 vs 89.86\% in 139His), suggesting that different genetic backgrounds may cause this discrepancy or that different populations may have different linkage disequilibrium patterns. A polymorphism may be in close linkage with another nearby causal variant in one ethnic population but not in another(Yu et al., 2010). The EPHX1 gene Tyr113His and His139Arg polymorphisms may be in close linkage with different nearby causal variants in different populations. On the other hand, in our subgroup analyses by ethnicity, the His139Arg polymorphism showed significant heterogeneous associations with EC across different ethnic groups, with 139His in Chinese being completely at odds with that in Canadians, suggesting that this polymorphism may have a pleiotropic role in the pathogenesis of EC or interact with other genetic and environmental 
factors. However, considering the relatively small sample sizes in Chinese and Canadians, we suggest that confirmation in large, well-designed studies is critical.

Despite the clear strengths of our study, including relatively large sample sizes and robustness of statistical analyses, interpretation of our current study, however, should be viewed in light of several technical limitations. First, all included studies were case-control in design, which precludes us to make inference on causality. Second, because only published studies were retrieved and articles in languages other than English were not included, publication bias might have occurred, even though our funnel plots and statistical tests indicated no observable bias. Third, our results were based on unadjusted estimates. It seems likely that the EPHXI gene Tyr113His and His139Arg polymorphisms may not make a significant contribution to risk prediction in EC patients individually, but whether the polymorphisms integrated with other risk factors will enhance the prediction requires additional research. Thus, a more precise analysis should be conducted with individual data, which would allow for adjustment by other co-variables such as age, gender, lifestyle, and other genetic factors.

Taken together, we extended previous individually underpowered studies by suggesting that EPHX1 gene Tyr113His and His139Arg may not be a potential candidate for the development of EC. Also, our observations leave open the question of heterogeneous effect of $113 \mathrm{Tyr}$ and 139 His allele across different ethnic populations. Nevertheless, for practical reasons, we hope that this study will not remain just another endpoint of research but rather will provide the background data for further investigations on the association of EPHXI gene with EC.

\section{REFERENCES}

Casson AG, Zheng Z, Chiasson D, MacDonald K, et al. (2003). Associations between genetic polymorphisms of Phase I and II metabolizing enzymes, p53 and susceptibility to esophageal adenocarcinoma. Cancer Detect. Prev. 27: 139-146.

Casson AG, Zheng Z, Porter GA and Guernsey DL (2006). Genetic polymorphisms of microsomal epoxide hydroxylase and glutathione S-transferases M1, T1 and P1, interactions with smoking, and risk for esophageal (Barrett) adenocarcinoma. Cancer Detect. Prev. 30: 423-431.

Cohn LD and Becker BJ (2003). How meta-analysis increases statistical power. Psychol. Methods 8: $243-253$.

Dura P, Bregitha CV, Te Morsche RH, Roelofs HM, et al. (2012). EPHX1 polymorphisms do not modify esophageal carcinoma susceptibility in Dutch Caucasians. Oncol. Rep. 27: 1710-1716.

Harrison DJ, Hubbard AL, MacMillan J, Wyllie AH, et al. (1999). Microsomal epoxide hydrolase gene polymorphism and susceptibility to colon cancer. Br. J. Cancer 79: 168-171.

Hassett C, Aicher L, Sidhu JS and Omiecinski CJ (1994a). Human microsomal epoxide hydrolase: genetic polymorphism and functional expression in vitro of amino acid variants. Hum. Mol. Genet. 3: 421-428.

Hassett C, Robinson KB, Beck NB and Omiecinski CJ (1994b). The human microsomal epoxide hydrolase gene (EPHX1): complete nucleotide sequence and structural characterization. Genomics 23: 433-442.

Hemminki K, Lorenzo BJ and Forsti A (2006). The balance between heritable and environmental aetiology of human disease. Nat. Rev. Genet. 7: 958-965.

Higgins JP and Thompson SG (2002). Quantifying heterogeneity in a meta-analysis. Stat. Med. 21: 1539-1558.

Higgins JP, Thompson SG, Deeks JJ and Altman DG (2003). Measuring inconsistency in meta-analyses. BMJ 327: 557-560.

Hiyama T, Yoshihara M, Tanaka S and Chayama K (2007). Genetic polymorphisms and esophageal cancer risk. Int. J. Cancer 121: 1643-1658.

Ihsan R, Chattopadhyay I, Phukan R, Mishra AK, et al. (2010). Role of epoxide hydrolase 1 gene polymorphisms in esophageal cancer in a high-risk area in India. J. Gastroenterol. Hepatol. 25: 1456-1462.

Jain M, Tilak AR, Upadhyay R, Kumar A, et al. (2008). Microsomal epoxide hydrolase (EPHX1), slow (exon 3, 113His) and fast (exon 4, 139Arg) alleles confer susceptibility to squamous cell esophageal cancer. Toxicol. Appl. Pharmacol. 230: 247-251.

Lancaster JM, Brownlee HA, Bell DA, Futreal PA, et al. (1996). Microsomal epoxide hydrolase polymorphism as a risk 
factor for ovarian cancer. Mol. Carcinog. 17: 160-162.

Lin YC, Wu DC, Lee JM, Hsu HK, et al. (2006). The association between microsomal epoxide hydrolase genotypes and esophageal squamous-cell-carcinoma in Taiwan: interaction between areca chewing and smoking. Cancer Lett. 237: 281-288.

Sims P, Grover PL, Swaisland A, Pal K, et al. (1974). Metabolic activation of benzo(a)pyrene proceeds by a diol-epoxide. Nature 252: 326-328.

Wang LD, Zheng S, Liu B, Zhou JX, et al. (2003). CYP1A1, GSTs and mEH polymorphisms and susceptibility to esophageal carcinoma: study of population from a high- incidence area in north China. World J. Gastroenterol. 9: 1394-1397.

Wang Z, Tang L, Sun G, Tang Y, et al. (2006). Etiological study of esophageal squamous cell carcinoma in an endemic region: a population-based case control study in Huaian, China. BMC Cancer 6: 287.

Xing D, Tan W and Lin D (2003). Genetic polymorphisms and susceptibility to esophageal cancer among Chinese population (review). Oncol. Rep. 10: 1615-1623.

Yu K, Zhang J, Zhang J, Dou C, et al. (2010). Methionine synthase A2756G polymorphism and cancer risk: a metaanalysis. Eur. J. Hum. Genet. 18: 370-378.

Zhang JH, Jin X, Li Y, Wang R, et al. (2003). Epoxide hydrolase Tyr113His polymorphism is not associated with susceptibility to esophageal squamous cell carcinoma in population of North China. World J. Gastroenterol. 9: 2654-2657. 